\title{
Pseudartroses e atrasos de consolidação de fracturas do rádio distal. Análise de 8 casos
}

\author{
J.C. Botelheiro(1), S. Silvério ${ }^{(2)}$ \\ UNIDADE DE CIRURGIA DA MÃo \\ Hospital de Sant'Ana, Parede; Hospital dos Lusíadas, Lisboa; Portugal \\ (1) Assistente Hospitalar GRadUAdo DE ORTOPEDIa \\ (2) Assistente Hospitalar DE ORTOPEDIa
}

Correspondencia:

Dr. José Carlos Botelheiro

Hospital de Sant'Ana

Rua de Benguela, s/n

2775 Parede, Portugal

e-mail: jcbotelheiro@gmail.com

\begin{abstract}
As pseudartroses e atrasos de consolidação são complicações muito raras das fracturas do rádio distal. Os Rx dinâmicos são o método essencial de confirmação diagnóstica.

Foram operados nos últimos 11 anos 8 casos. Em sete a cabeça do cúbito foi excisada e usada para preenchimento da pseudartrose.Os métodos de fixação usados foram em seis casos os fios Kirschner e em dois uma placa palmar. Só num caso, que já tinha tido uma operação de Darrach préviamente, foi utilizado o enxerto ilíaco.

Todos consolidaram, com uma mobilidade e força de preensão de pelo menos $50 \%$ do lado oposto. Num doente, que recusou a extracção dos fios Kirschner observou-se uma rotura do tendão do extensor pollicis longus; foi reoperado (transferência tendinosa com extensor indicis propius) tendo-se obtido um bom resultado final.
\end{abstract}

Palavras-chave: pseudartrose rádio distal.
Pseudarthrosis and delayed unions are very rare complications of distal radius fractures. Dynamic $\mathrm{X}$-rays are very usefull for the diagnosis.

Eight cases were operated in the last 11 years. In 7 the head of the cubitus was excised and used as a graft to the pseudarthrosis, fixed in 6 with Kirschner wires and in 2 with a volar plate. In one case, that had a previous Darrach operation, iliac bone graft was used.

All went to union, with a wrist mobility and grip strength of at least $50 \%$ of the contraleral side. One patient didn't come back to remove the Kirschner wires and had a rupture of the extensor pollicis longus tendon - a transfer of the extensor indicis propius was made with a good final result.

Key words: distal radius nonunion. 


\section{INTRODUÇÃO}

$\Lambda$ $\mathrm{s}$ fracturas distais do rádio ocorrem em osso esponjoso, pelo que as pseudartroses e atrasos de consolidação são complicações muito raras ${ }^{1,2}$, de tal forma que 2 grandes revisões desse tipo de fracturas nem as mencionam $^{3,4}$. Tão pouco se conhecem as razões dessas raras ocorrências, embora se mencionem a distracção excessiva com placas ou fixadores externos, fracturas concomitantes do cúbito e factores gerais, como o tabaco e o alcoolis$\mathrm{mo}^{2,5,6}$. A falta de consolidação até aos 4 meses é geralmente considerada um atraso de consolidação e após os 6 uma pseudartrose ${ }^{5}$.

O primeiro caso mencionado na literatura foi operado com um enxerto tibial suturado com catgut ao rádio e aos metacárpicos e seccionado depois distalmente para ter alguma mobilidade do punho ${ }^{7}$. A maior série desta patologia publicada na literatura internacional, juntando os casos de 3 centros de cirurgia da mão da Europa e EUA', é de 26 caso apenas.

O diagnóstico pode não ser óbvio e as radiografias dinâmicas são geralmente consideradas muito úteis?.

As cirugias aconselhadas nos poucos artigos sobre o assunto são o tratamento da pseudartrose com placas e enxerto ilíaco intercalar ${ }^{5,8,9}$ e a artrodese do punho ${ }^{1,2}$, esta quando o fragmento distal tiver menos de $5 \mathrm{~mm}$ acima da superfície $\operatorname{articular}^{2}$. Cirurgias múltiplas são frequentes ${ }^{5,8,9}$.

\section{MATERIAL E MÉTODO}

Nesta pequena série, só 3 doentes eram do sexo masculino e a idade oscilava entre os $24 \mathrm{e}$ os 72 anos, sendo os detalhes apresentados no quadro junto (Tabela I) e na Discussão. De notar que 4 casos eram acidentes laborais (referenciados HPO e HLU).

\section{RESULTADOS}

Quanto aos resultados, de notar que se obteve a consolidação em todos os casos com una única cirurgia.

A mobilidade final obtida foi sempre superior a $80^{\circ}$ de flexao-extensão do punho; e pronosupinasão quase sempre completa.

A força de preensão após a cirurgia variou entre os $40 \%$ e os $90 \%$ do lado contrário, semre muito superior á pré-operatória. Dois casos laborais apresentavam instabilidade cubital, dolorosa num.

Tabla I - Principals dados dos casos operados

\begin{tabular}{|c|c|c|c|c|c|c|c|c|c|c|c|}
\hline Caso & Ident & Idade & Sexo & Acid. & Trat. & Cirurgia & Técnica & Extr fks & Fl-ext & Pron-supin & Preensão \\
\hline 1 & TLO & 24 & $M$ & $\begin{array}{c}12-2000 \\
\text { (auto) }\end{array}$ & Cirurg & $4-2001$ & $\begin{array}{c}\text { fks } \\
\text { Darrach }\end{array}$ & $\begin{array}{c}2002 \\
\text { eip-epl }\end{array}$ & $80^{\circ}$ & $90^{\circ}-45^{\circ}$ & $80 \%$ \\
\hline 2 & $\begin{array}{c}\text { HOSA } \\
122339\end{array}$ & 65 & $M$ & $\begin{array}{c}\text { 7-2003 } \\
\text { (auto) }\end{array}$ & Fix.ext. & $5-2004$ & $\begin{array}{c}\text { fks } \\
\text { Darrach }\end{array}$ & 3-2005 & $90^{\circ}$ & $\mathrm{N}$ & $60 \%$ \\
\hline 3 & HPO & 60 & $\mathrm{~F}$ & $3-2004$ & Conserv. & $6-04$ & $\begin{array}{c}\text { fks } \\
\text { Darrach }\end{array}$ & $10-04$ & $\mathrm{~N}$ & $\mathrm{~N}$ & - \\
\hline 4 & $\begin{array}{c}\text { HLU } \\
132638\end{array}$ & 61 & $\mathrm{~F}$ & $\begin{array}{l}\text { 4-09 } \\
\text { (auto) }\end{array}$ & Fix.ext. & $7-09$ & $\begin{array}{l}\text { Placa } \\
\text { Darrach }\end{array}$ & Algodistr. & $80^{\circ}$ & $\mathrm{N}$ & - \\
\hline 5 & $\begin{array}{c}\text { HLU } \\
176929\end{array}$ & 72 & $\mathrm{~F}$ & $1-2010$ & Conserv & $4-10$ & $\begin{array}{c}\text { Fks } \\
\text { Darrach }\end{array}$ & $\begin{array}{c}\text { 7-10 } \\
\text { Inst.cub. }\end{array}$ & $90^{\circ}$ & $\mathrm{N}$ & - \\
\hline 6 & $\begin{array}{c}\text { HOSA } \\
174273\end{array}$ & 71 & $F$ & 8-09 & $\begin{array}{c}\text { Fks } \\
\text { Darrach }\end{array}$ & $5-11$ & $\begin{array}{c}\text { Placa } \\
\text { Enx.liaco }\end{array}$ & & $80^{\circ}$ & $\mathrm{N}$ & $40 \%$ \\
\hline 7 & $\begin{array}{c}\text { HLU } \\
32929\end{array}$ & 50 & $\mathrm{~F}$ & $6-11$ & Conserv & $11-11$ & $\begin{array}{c}\text { Fks } \\
\text { Darrach }\end{array}$ & $3-12$ & $100^{\circ}$ & $\mathrm{N}$ & Dores \\
\hline 8 & $\begin{array}{c}\text { HOSA } \\
179754\end{array}$ & 72 & $M$ & $8-11$ & Conserv & $1-12$ & $\begin{array}{c}\text { Fks } \\
\text { Darrach }\end{array}$ & $5-12$ & $90^{\circ}$ & $\mathrm{N}$ & $90 \%$ \\
\hline
\end{tabular}




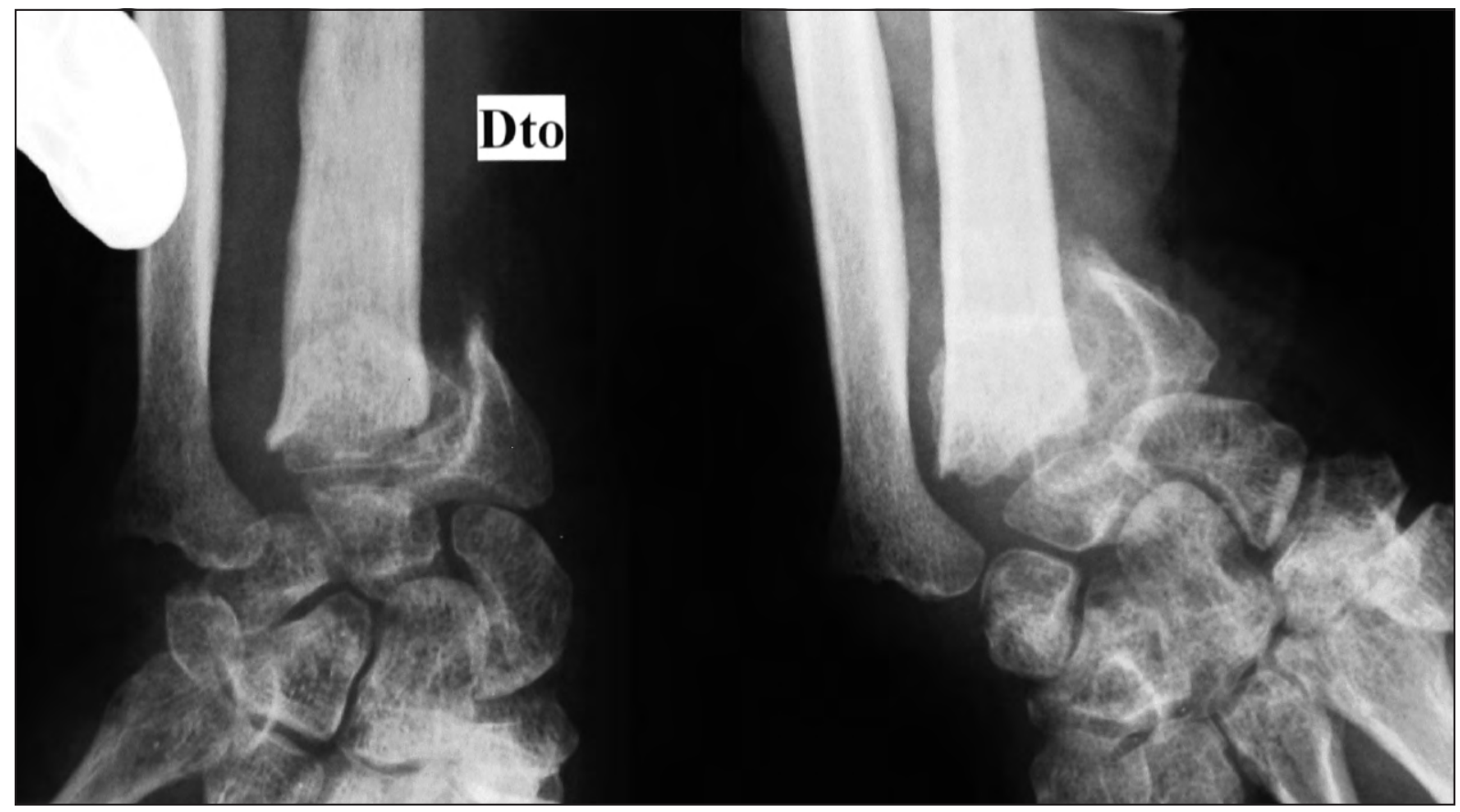

Figura 1. Mobilidade clara nas radiografias dinâmicas da pseudartrose do caso 2.

Apresenta-se também detalhadamente um caso com as suas radiografias:

Caso 2: Doente do sexo masculino, nascido em 1938. Fractura distal do rádio direito em acidente de viação em julho 2003 tratada com fixador externo durante 8 semanas na Holanda, resultando pseudartrose evidente com fragmento distal muito pequeno, para a qual foi proposta artrodese do punho (Figura 1). Operado no Hospital de Sant'Ana a 7-5-2004 - excisão distal de cúbito muito longo, cujo enxerto esponjoso foi utilizado para preenchimento do foco de pseudartrose, abordado por via dorsal separada e fixado com 2 fios de Kirschner. Imobilização post-operatória com tala gessada em U, substituida às 2 semanas por tala antebraquio-palmar amovível até às 10 semanas. Obteve-se consolidação sólida (Figura 2), com uma flexão-extensão do punho $90^{\circ}$, uma prono-supinação quase normal e uma força de preensão final de $60 \%$.

Extracção dos fios Kirschner justa-ósseos a 4-3-2005.

\section{DISCUSSÃO}

Como se mencionou inicialmente, estas pseudartroses são muito raras e não se sabe exacta- mente porque ocorrem. Nesta pequena série 5 casos são de alta energia, dois deles tratados com fixador externo (casos 2 e 4 ). Nenhum doente tinha hábitos etílicos ou tabágicos pronunciados.

Apenas 2 casos eram verdadeiras pseudartroses, com mais de 6 meses de evolução (casos 2 e 6), devendo os outros ser considerados atrasos de consolidação, com um tempo médio de evolução de 4 meses. Optou-se no entanto nestes também pela cirurgia dados os riscos dessa imobilização prolongada - não consolidação e rigidez do punho - e pelo comprimento excessivo do cúbito que obrigaria mais tarde a correcção cirúrgica.

Já o seu tratamento foi bastante homogéneo - tratamento da pseudartrose - ao contrário das séries publicadas que todas incluem casos de artrodese do punho. A osteossíntese foi efectuada com 2 fios Kirschner nos caso mais distais - seis - e com placa palmar nos outros dois (casos 4 e 6). Dos 8 doentes operados, em 7 foi usado enxerto esponjoso da cabeça do cúbito, excisada em 6 por estar procidente e num por se encontrar também em pseudartrose (caso 4). Só num caso, previamente submetido a operação de Darrach de difícil justificação, se utilizou enxerto do ilíaco.

Nos casos com fragmento distal muito pequeño optou-se pela fixação da pseudartrose com fios Kirschner na melhor posição possível, evi- 


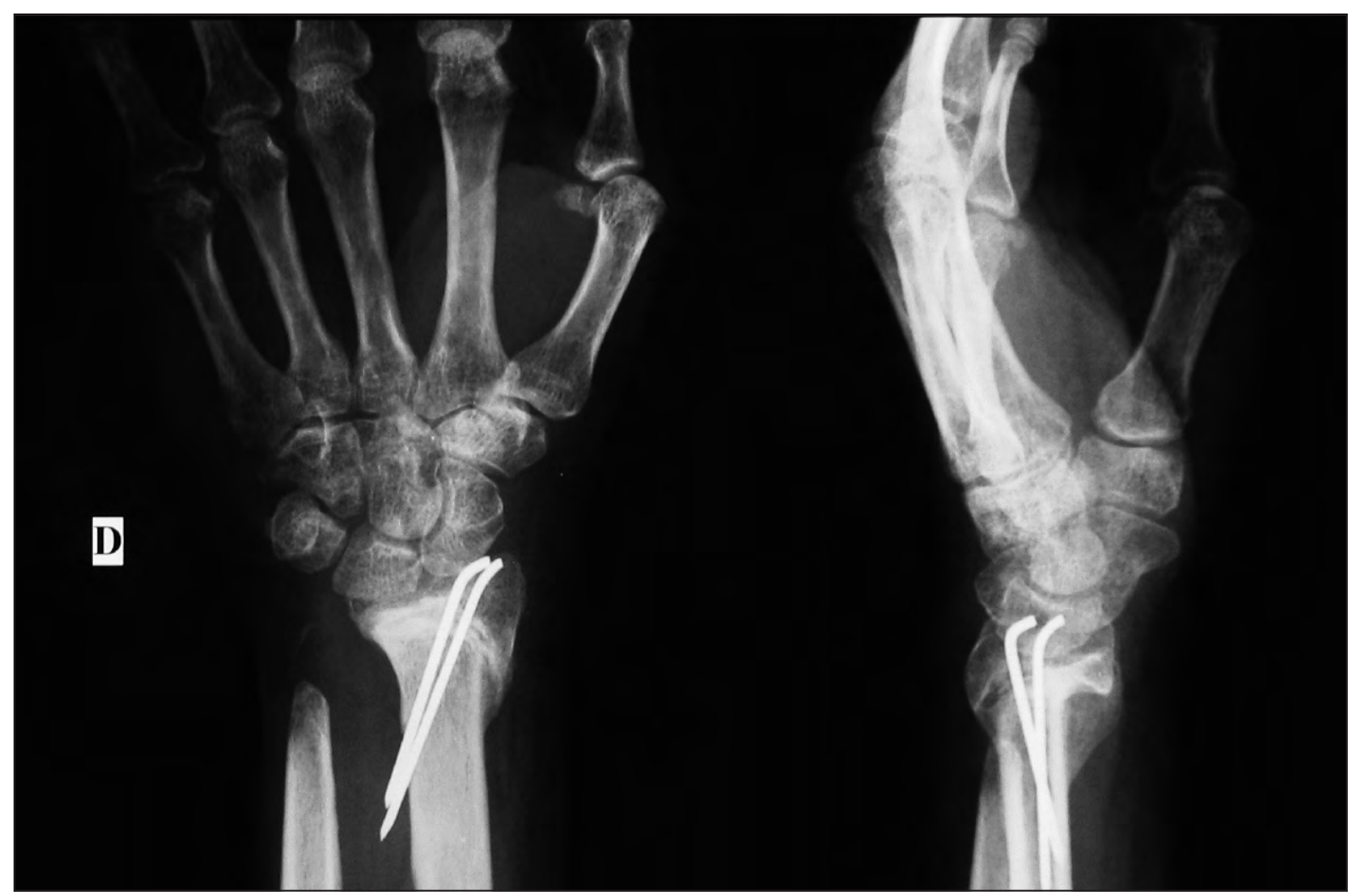

Figura 2. Consolidação post-operatória do mesmo caso.

tando a artrodese do punho e preservando assim um arco de mobilidade útil.

A excisão distal do cúbito fornece enxerto ósseo suficiente, resolve os problemas de incongruência rádio-cubital distal e da impacção cúbito-cárpica; desta forma, os possíveis inconvenientes da operação de Darrach tornam-se num mal menor. E evita-se assim o alongamento do rádio com enxerto ilíaco intercalar, de difícil e arriscada execução técnica com fragmentos distais pequenos e osteoporóticos mesmo utilizando um distractor externo. De qualquer forma, em todos os casos, o ilíaco homolateral foi preparado para possível utilização.

\section{BIBLIOGRAFIA}

1. Smith VA, Wright TW. Non-union of the distal radius. J Hand Surg $\mathrm{Br}, 1999 ; 24:$ 601-4.

2. Segalman KA, Clark GL. Un-united fractures of the distal radius:a report of 12 cases. J Hand Surg Am, 1998; 23: 914-9.

3. Bacorn RW, Kurtke JF. Colle's fracture: a study of two thousand cases from the New York Workmen's Compensation Board. J Bone Joint Surg Am, 1953; 35: 643-58.

4. Camelot C, Ramaré S, Lemoine J, Saillant G. Traitement orthopédique des fractures de l'extrémité inférieure du radius selon Judet: à propos de 280 cas. Rev Chir Orthop, 1998; 84: 124-35.

5. Fernandez DL, Ring D, Jupiter JB. Surgical management of delayed union and nonunion of distal radius fractures. J Hand Surg Am, 2001; 26: 201-9.

6. Yañez J, Castellano I, Mendoza M. Seudoartrosis de las fracturas del extremo distal del radio. Acta Ortop Gallega, 2011; 7: 93-9.
7. Hamada G. Extra-articular graft for non-union in Colles' fracture. J Bone Joint Surg, 1944; 26 : 833-5.

8. Prommersberger KJ, Fernandez DC, Ring D, Jupiter JB, Lanz MB. Non-union of distal radius fractures - 26 cases. Chir Main, 2002; 21: 113-23.

9. Harper WM, Jones JM. Nonunion of Colles' fracture: report of two cases. J Hand Surg Br, 1990; 15: 121-3. 